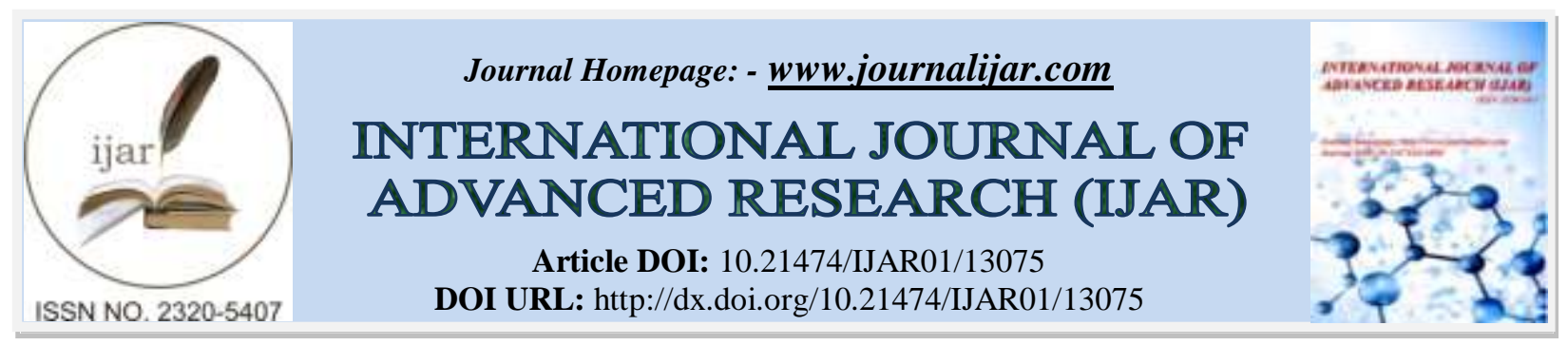

RESEARCH ARTICLE

\title{
SEVEREULCERATIVE COLITIS ASSOCIATED WITH COOMBS POSITIVE AUTOIMMUNE HEMOLYTIC ANEMIA TREATED WITH INFLIXIMAB
}

Busbaih, Albatool Abdulmajeed, Hodaya, Ghufran Ali, Busbaih and Jawad Salman

\section{Manuscript Info}

Manuscript History

Received: 25 April 2021

Final Accepted: 28 May 2021

Published: June 2021

Key words:-

Autoimmune Hemolytic Anemia

(AIHA), Ulcerative Colitis, Infliximab

\section{Abstract}

Autoimmune hemolytic anemia is rarely associated with ulcerative colitis, and counts only for $0.2-0.7 \%$ of adult patient.Coombs positive autoimmune hemolytic anemia which account for only $1.82 \%$ in patient with ulcerative colitis without any hemolytic evidence(1). Here we report a case of sever ulcerative colitis associated with Coombs positive autoimmune hemolytic anemia (AIHA) in response to Infliximab.

Copy Right, IJAR, 2021,. All rights reserved.

\section{Introduction:-}

Autoimmune hemolytic anemia is rarely associated with ulcerative colitis, The incidence of both condition simultaneously is only $0.2-0.7 \%$ in adult patient, so it is a rare complication that is presented by having a positive coombs test which account for only $1.82 \%$ in patient with ulcerative colitis without any hemolytic evidence [1].

The reason or the etiology is not yet understood. It is likely due to multifactorial processes including the production of anti-erythrocytes antibodies by the colon, that explains the therapeutic effect of hemolysis after colectomy in ulcerative colitis patient [1].

Monoclonal antibodies like Infliximab has potent of anti-inflammatory activity, which helps as a therapeutic agent for Rheumatoid arthritis, inflammatory bowel disease, and many other autoimmune diseases [2].

Here we report a case of severe ulcerative colitis associated with Coombs positive autoimmune hemolytic anemia (AIHA) in response to Infliximab

\section{Case Presentation}

A 35 years old Saudi male diagnosed as a case of ulcerative colitis on 2011 by colonoscopy which showed mild to moderate left side colitis when he presented with bloody diarrhea 6-8 time /day for 4 month, Weight loss about $5 \mathrm{~kg}$ in the same period. He was started on Mesalazine enema foam. One week after the start of Mesalazine , the patient presented back with signs and symptoms of anemia and hemolysis ( $\mathrm{Hb} 5 \mathrm{~g} / \mathrm{dl})$

(Jaundice - high Indirect bilirubin - high LDH- high retculositosis - Reduced Haptoglobulin ). Along with a positive Coombs test was, which suggest an autoimmune hemolytic anemia, with exclusion of other etiologies. Patient was treated with predinsolone $60 \mathrm{mg}$ and azathioprine $150 \mathrm{mg}$.

Patient symptoms improved with azathioprine and steroid. However, upon tapering down the steroid dose his symptoms deteriorated and he required re-introducing steroid. He was then kept on Steroid 7.5 mg, Azathioprine 
150mg, Mesalazine, Calcium, Vitamin D and Folic acid.

The patient presented back on 2013 to the hospital with symptom of anemia, fatiguability, tiredness, and palpitation. He also reported a proximal myopathy with decrease activity.

He had his baseline bowel movement of 2-3 times per day with occasional blood in stool.

He was complaint to his medication.

On examination, the patient looked sick, cachectic, and pale. He wasn't jaundice.

Vital signs were normal and his exam was unremarkable apart from proximal upper and lower weakness, otherwise his neurological exam was normal.

\section{Investigations:}

wbc: $3.6 \times 1000 / \mathrm{mm}^{\wedge} 3$

$\mathrm{Hb}: 8.3 \mathrm{~g} / \mathrm{dl}$

platelet: $660 \times 1000 / \mathrm{mm}^{\wedge} 3$

MCV: $79 \mathrm{fl}$

MCH: 33 pg

RFT,LFT and electrolytes : normal

Total Protein $44 \mathrm{mg}$

Albumen: $14 \mathrm{~g} /$ liter

Ca: $1.96 \mathrm{mg} / \mathrm{dl}$

ESR: $120 \mathrm{~mm} / \mathrm{hr}$

CRP: $8.3 \mathrm{mg} / \mathrm{dl}$

Coombs test direct; ++++

B12 level and Folate level are normal

Patient's medications were adjusted again to predinsolone $50 \mathrm{mg}$, Azathioprine $100 \mathrm{mg}$, and mesalazine $1 \mathrm{gm}$ PO daily.

He had colonoscopy and it revealed the following: [pic-1]

Very severe diffuse inflammation with multiple variable size of pseudo polyps with narrow lumen at $70 \mathrm{~cm}$. The scope pass up to T-colon $70 \mathrm{~cm}$ due to narrow lumen.

Multiple biopsies were taken and it showed chronic active colitis.

His CT Abdomen showed the following;

Diffuse thickened wall of Recto-sigmoid as well as transverse and descending colon with increased pericolic vascularity (Comb sign), multiple polyp like structures seen at rectosigmoid region mostly represent pseudopolyp, it is seen associated with cast like intracolonic structure along descending colon, with target like appearance on axial images and contrast seen interposed between it and colonic wall, mostly represent sloughed thickened mucosa.

The final diagnoses was made to be severe ulcerative colitis associated withautoimmune hemolytic anemia. Regarding the therapeutic options we were in front of two approaches after the failure of steroid and immunosuppressant agent in the therapy of both Ulcerative colitis and AIHA. We decided to institute a biological agent therapy instead of colectomy.

Review of the recent literature shows similar condition in which Anti-TNF medication like infilixmab was given to the patient with autoimmune hemolytic anemia and severe ulcerative colitis. The result was satisfactory, Infliximab is a good option to start therapy with before going for surgical options like splenectomy and/or colectomy [1]. 
Our patient was started on Infliximab infusion every 8 weeks and he continued on mesalazine 1 gm Po daily. The patient showed significant clinical and biochemical improvement. [Table-1]

The patient repeated scope confirmed patient is on remission.[pic-1-2]

[Table-1]

\begin{tabular}{|c|c|c|c|c|}
\hline & 5/10/2013 & $29 / 11 / 203$ & $25 / 12 / 200$ & $7 / 2 / 2041$ \\
\hline Wectoogimm's & 3.7 & 16 & 7 & 5 \\
\hline He gla & 7.7 & & $\pi$ & 15 \\
\hline gisteles $7000 \mathrm{~mm} 3$ & 660 & & 300 & 210 \\
\hline Total Pioten ing & 44 & & 58 & 75 \\
\hline Aitumen ol & 14 & & 44 & 45 \\
\hline ESR mmint & 120 & & 46 & 13 \\
\hline CRP mgidk: & 8.1 & & 2.7. & 0.5 \\
\hline wt & $52 \mathrm{~kg}$ & & & $77 \mathrm{~kg}$ \\
\hline
\end{tabular}

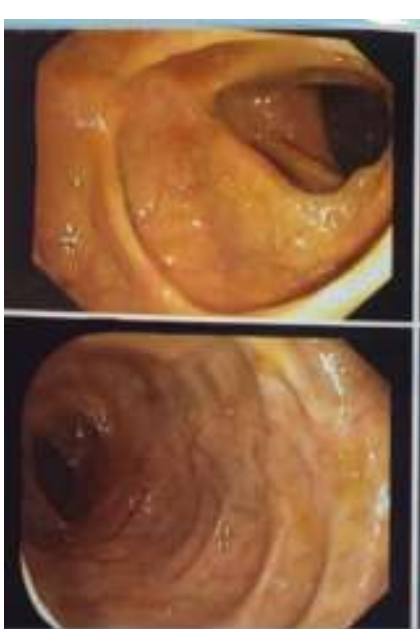

[Pic-1]

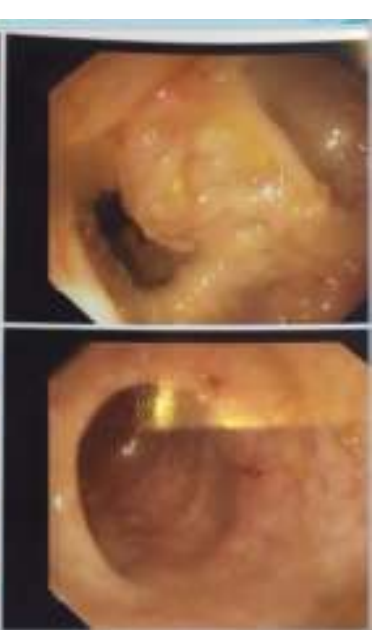

$\Rightarrow$
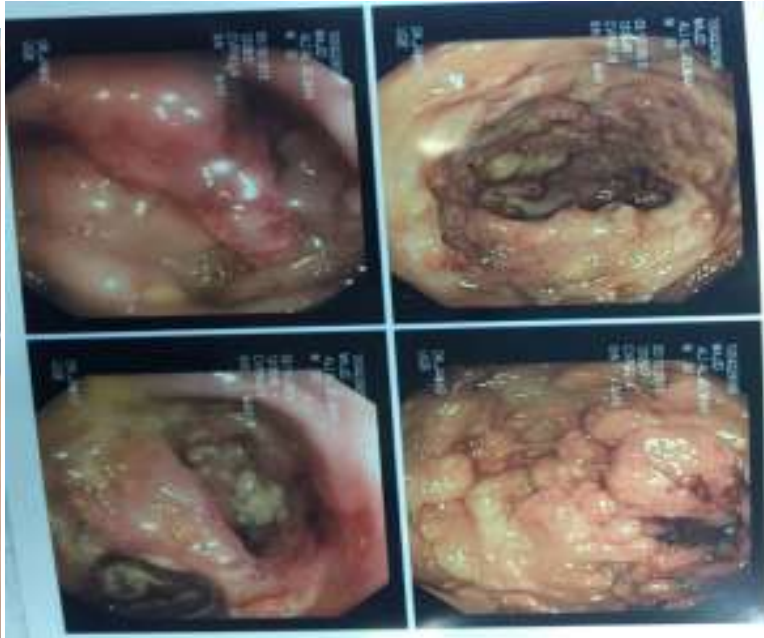

[Pic-2]

\section{Discussion:-}

Infliximab is biological drugs that is work by blocking the action of tumor necrosis factor-A to decrease or slow the body immunity. Monoclonal antibodies like infliximab has potent anti-inflammatory activity, which helps as a therapeutic agent for Rheumatoid arthritis, inflammatory bowel disease, and many other autoimmune diseases [2].

There are many side effects associated with the Infliximab in general and in infusion specifically includes, an infusion reaction, which can be manifested by swelling or pain on the site of injection or general reaction.

Flushing, shortness of breath, risk of infections, pancytopenia and hematological malignancies. [2,3].

Ulcerative colitis (UC) is an inflammatory bowel disease that affects the colon mainly, while autoimmune hemolytic anemia is a rare complication of such disease and it represent only minority of patient,

Ulcerative colitis mainly respond to steroid as a first line management, and shows a remission rate in $21 \%-50 \%$ of cases [2]. But unfortunately this line of management did not work with our patient; going back to the etiology of having a Coombs positive autoimmune hemolytic anemia associated with ulcerative colitis is not fully understood. There are multiple theories that may explain the condition. The first one is stated by the provocation of the immune system by chronic inflammatory bowel disease, or induced by anti-erythrocytes antibody. The second explanation is by the cross- reaction of antibodies between both red blood cells and the colon [4]. 
In our cases, the patient didn't show any improvement with the immunosuppressant agent and systemic steroid. After reviewing the literature of similar condition which suggest the use of biological agent [5].

Other option was Rituximab which was reviewed in the literature for autoimmune hemolytic anemia [6]. We found one study that used Rituximab for autoimmune hemolytic anemia and severe ulcerative colitis [7].

On that base, we decided to use Infilixmab as induction therapy and maintenance therapy. The patient had good response which can be explained by the healing of the mucosal layer that will suppress the expression of antigens that may cross-react with antigen of RBCs, that is suggested by the above mentioned theories [8].

\section{Conclusion:-}

Here, we concluded that the usage of biological agent like infliximab, before considering surgical approaches, may shows a great response for both conditions, therefore, such case can open a huge opportunities for more researches about using infliximab as a new therapeutic agent for both ulcerative colitis and AIHA especially after failure of controlling the UC with the immunosuppressant agents or for AIHA with any other disease other than inflammatory bowel diseases.

\section{References:-}

1. Pozzi, E; Micheli, A; Bronzini, F; Brondello, C; Lionetti, P Coombs-positive: Autoimmune Haemolytic Anemia in Ulcerative Colitis: Spectrum of Same Disease?. Journal of Pediatric Gastroenterology and Nutrition: November. 2006, 43:49-1590. https://www.dldjournalonline.com/article/S1590-8658(06)00401-4/abstract

2. Paul Rutgeerts, Ph.D., William J. Sandborn, et al.: Infliximab for Induction and Maintenance Therapy for Ulcerative Colitis. N Engl J Med 2005;353: 2462-76. Fazia A. Mir, Alhareth Al Juboori, Jack D. Bragg.

3. Autoimmune hemolytic anemia associated with infliximab infusion in ulcerative colitis. North Clin Istanb. 2018, 5:64-66. https://www.ncbi.nlm.nih.gov/pmc/articles/PMC5864712/

4. Autoimmune Haemolytic Anaemia associated with Ulcerative Colitis,CASE REPORT , JIACM 2002; 3(2): 198-201. Eduardo Leo Carnerero MD, Sami Aoufi MD, Isabel Montero Cuadrado MD, Pablo Herrera Martin MD \& Jose Manuel Herrera Justiniano MD: https://gavinpublishers.com/articles/case-report/Journal-ofDigestive-Diseases-and-Hepatology/autoimmune-hemolytic-an....

5. 2371 (2009) Suárez A1, San Román FS, Rodríguez M, Riestra S, Navascués CA: Autoimmune Hemolytic Anemia Associated With Ulcerative Colitis: Response to Infliximab,The. American Journal of Gastroenterology, volume 104, pages. 1994, 85:471-3.

6. Autoimmunity associated with anti-tumor necrosis factor alpha treatment in Crohn's disease: a prospective cohort study. Gastroenterology. 2003, 125:32-9.

7. Rituximab in the treatment of autoimmune haematological disorders. Br J Haematol. 2008, 1111:1365-2141. https://onlinelibrary.wiley.com/doi/full/10.1111/j.1365-2141.2008.07054.x

8. Successful treatment of steroid resistant ulcerative colitis associated with severe autoimmune hemolytic anemia with oral microemulsion cyclosporin— a brief case report. Am J Gastroenterol. 2003, 98:1207-8. 